WER BRAUCHT EINE AUFFRISCHIMPFUNG?

\section{Neues Merkblatt zu Polio}

Europa ist laut WHO seit Juni 2002 poliofrei, der amerikanische Kontinent bereits seit 1994. Trotzdem müssen Sie bei bestimmten Patienten nach wie vor an eine Auffrischimpfung denken, z. B. bei Reisenden in Regionen mit Infektionsrisiko, aber auch bei Aussiedlern, Flüchtlingen und Asylbewerbern, die in Gemeinschaftsunterkünften leben und aus Gebieten mit Poliorisiko kommen. Weitere aktuelle Informationen zur Poliomyelitis und Kontaktstellen, an die Sie sich bei Fragen wenden können, finden Sie im Merkblatt für Ärzte, das vom Robert Koch-Institut im Januar 2010 aktualisiert wurde (www.rki.de).

KE =

\section{SCHUBRATE REDUZIERT}

\section{Stillen gegen MS}

Wöchnerinnen mit schubförmiger multipler Sklerose (MS) sollten Sie zum Stillen ermuntern, denn hierdurch wird ihre Schubrate reduziert. Zu diesem Ergebnis kommt eine Studie mit 32 schwangeren MS-Patientinnen, die während jedes Trimenons sowie bis zwölf Monate post partum untersucht wurden.

$52 \%$ der Frauen entschieden sich, nicht zu stillen, oder begannen innerhalb der ersten zwei Monate mit regelmäßigem Zufüttern. Von diesen erlitten $87 \%$ einen Schub innerhalb der ersten zwölf Monate

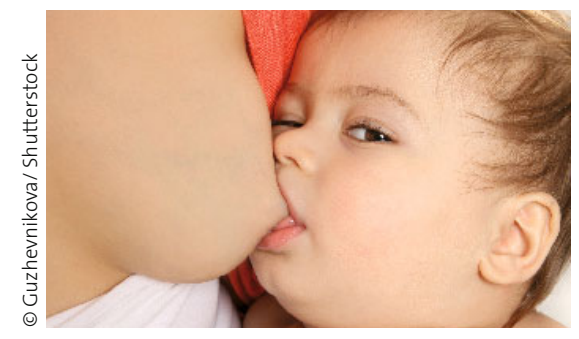

Lange Stillzeit, lange schubfrei.

post partum. Im Vergleich dazu zeigten nur $36 \%$ der Frauen, die für mindestens zwei Monate stillten, einen Schub in den ersten zwölf Monaten $(p=0,02)$. Mit diesem Ergebnis wird die bisherige Empfehlung infrage gestellt, wonach MS-Patientinnen rasch abstillen sollten, um ihre immunmodulatorische Therapie möglichst bald wieder aufnehmen zu können.

MO -

KREUZSCHMERZEN

\title{
Aufwendige Diagnostik, wenig Beratung
}

Akute Rückenschmerzen ohne Hinweis auf eine Nervenwurzelkompression oder eine gravierende Erkrankung, verschwinden in der Regel auch ohne Behandlung innerhalb weniger Wochen. Als behandelnder Arzt sollte man die Patienten primär über die gute Prognose aufklären und ihnen nahelegen, sich nicht zu schonen, sondern körperlich aktiv zu bleiben.

In der Praxis sieht das leider häufig anders aus, wie eine Studie mit 3533 Patienten mit akuten Kreuzschmerzen zeigt: Nur jeder fünfte Patient wurde beraten. Häufiger als eine Beratung erhielten die Patienten sogar eine Überweisung zur bildgebenden Diagnostik - eine Maßnahme, die bei akutem Rückenschmerz ohne Warnsignale nicht empfohlen wird. Zur medikamentösen Therapie wurde den Patienten in $18 \%$ der Fälle Paracetamol verordnet (das als ers-

Jeder vierte Patient mit akuten Kreuzschmerzen erhält eine bildgebende Untersuchung. te Wahl empfohlen wird). $37 \%$ erhielten ein nicht steroidales Antirheumatikum und 20\% sogar Opioide.

Arch Intern Med 2010;170:271-277

\section{AKUTES KORONARSYNDROM}

\section{Gesunder Lebensstil zahlt sich schon nach sechs Monaten aus}

Gesunde Ernährung, Bewegung und Rauchverzicht sollten bei Patienten nach akutem Koronarsyndrom die gleiche Priorität haben wie die Pharmakotherapie. Durch Änderungen des Lebensstils können sie ihr kardiovaskuläres Risiko schon innerhalb von sechs Monaten reduzieren. Der Effekt ist additiv zu dem der üblichen medikamentösen Therapie.

Durch Rauchverzicht wird das Risiko eines erneuten Herzinfarkts in dieser Zeit in etwa halbiert. Ungefähr denselben Effekt erzielen Ernährungsumstellung plus
Sport. Dagegen haben Patienten, die unverändert rauchen, futtern und faul sind, im halben Jahr nach dem Ereignis ein fast viermal so hohes Risiko für Herzinfarkt, Schlaganfall oder Tod wie Patienten, die in jeder Hinsicht gesund leben, so die Ergebnisse einer Studie mit 18800 Patienten. Die Studie zeigt aber auch, was jeder Arzt aus Erfahrung weiß: Die meisten Patienten sind viel eher bereit, ein paar Tabletten zu schlucken, als ihren Lebensstil umzukrempeln.

BS -

Circulation 2010:121;750-758 